\title{
On Bipolar Possibility Fuzzy Soft Sets
}

\author{
Congcong Meng ${ }^{\text {a) }}$, Bo Hai ${ }^{\text {b) }}$ and Hailong $\mathrm{Yao}^{\mathrm{c}}$ \\ College of Electronic and Information Engineering, Lanzhou City University, Lanzhou 730070, China. \\ a) Corresponding author: mcc@1zcu.edu.cn \\ b) hb@1zcu.edu.cn \\ c)hailongyao@outlook.com
}

\begin{abstract}
The concept of bipolar possibility fuzzy soft sets is proposed and some basic operations on them are defined such as complement, extended union, extended intersection, restricted union, restricted intersection, "AND","OR". Then their properties are investigated. An application of bipolar possibility fuzzy soft sets in a decision-making problem with a general algorithm is presented at the end of this article.
\end{abstract}

Key words: Soft Set, Fuzzy Soft Set, Possibility Fuzzy Soft Set, Bipolar Soft Set, and Bipolar Possibility Fuzzy Soft Set.

\section{INTRODUCTION}

In our real life, there exist lots of uncertain, imprecise and vague problems in the fields of economics, engineering, environment and so on. Classical mathematical methods can solve the problems with determinacy or precision, but they may not successfully deal with the complicated problems with uncertainty. To deal with such problems, a number of theories have been proposed in an effective way, such as theory of probabilities [8], theory of fuzzy sets [18], theory of intuitionistic fuzzy sets [5], theory of vague sets [7], theory of interval mathematics [9], theory of rough sets [15], etc. However, all these theories have their inherent limitations as pointed out in [14]. To overcome these difficulties, Molotov [14] proposed the soft sets theory, which has become a completely new generic mathematical tool for modeling uncertainties.

Presently, the work on the soft set theory is making a great and rapid progress. Majid et al. [11, 12], Roy and Majid [16] have further studied the theory of soft sets and used this theory to solve some decision-making problems. Majid et al. [13] have also introduced the concept of fuzzy soft sets, a more general concept, which is a combination of fuzzy sets and soft sets and studied its properties. Zoo and Xiao [19] initiated soft sets into the incomplete environment. Alkhazaleh et al. [1] proposed the concept of soft multi sets as a generalization of soft sets. They also defined the concept of fuzzy parameterized interval-valued fuzzy soft sets [2] and applied it to the decision-making problems. Alkhazaleh and Saleh [3] presented the concept of possibility fuzzy soft sets where the possibility of each element in the universe is attached with the parameterization of fuzzy sets while defining a fuzzy soft set.

Dubois and Pride in [6] defined the role of polarity by saying that choices of people are usually caused by checking the good sides and the bad sides of alternatives separately, then they choose according to which sides are stronger. In other words, the judgments also possess an intrinsic positive or negative flavor, which is called a polarity. Bipolarity refers to an explicit handling of positive and negative sides of information. Shabbier and Nazi [17] introduced a new concept of bipolar soft sets, considering not only a chosen set of parameters but also an allied set of opposite meaning parameters. In 2000, Lee [10] initiated an extension of fuzzy sets named bipolar-valued fuzzy set. Aslam and Abdullah [4] gave a new concept of bipolar fuzzy soft sets combing the concept of bipolar fuzzy sets and soft sets afterwards. This paper aims to further generalize the concept of bipolar soft sets to a new soft set model named possibility bipolar fuzzy soft sets.

The rest of this paper is organized as follows. In Section 2, the notions of soft sets, fuzzy soft sets, possibility fuzzy soft sets, and bipolar soft sets are recalled. In Section 3, the concept of bipolar possibility fuzzy soft sets is 
proposed, some operations on bipolar possibility fuzzy soft sets and their properties are investigated. Section 4 introduces the concept of comparison tables, which are applied to decision making based on bipolar possibility fuzzy soft sets. Some illustrative examples are also employed to show that the method presented is not only reasonable but also more efficient in practical applications. Finally, Section 5 presents the conclusions.

\section{PRELIMINARIES}

In this section, the concepts of soft sets, fuzzy soft sets, possibility fuzzy soft sets, bipolar soft sets and so on are briefly reviewed. Further details could be found in [14, 13, 3, and 17]. Throughout this paper, unless otherwise stated, $\mathrm{U}$ refers to be an initial universe, E is a set of parameters, $\mathrm{P}(\mathrm{U})$ is the power set of $\mathrm{U}, I^{U}$ is the set of all fuzzy subsets of $U, \operatorname{PFSS}(\mathrm{U})$ is the set of possibility fuzzy soft set over $\mathrm{U}, \mathrm{A}, \mathrm{B}, \mathrm{C}$ are non-empty subsets of $\mathrm{E}$.

Definition 2.1(see [14]). A pair (F, A) is called a soft set over $\mathrm{U}$ where $\mathrm{F}$ is a mapping given by $F: A \rightarrow P(U)$.

In other words, a soft set over $\mathrm{U}$ is a parameterized family of subsets of the universe $\mathrm{U}$. For $\varepsilon \in A, F(\varepsilon)$ may be considered as the set of $\varepsilon$-elements of the soft set (F, A) or as the set of $\varepsilon$-approximate elements of the soft set (F, A).

Definition 2.2(see [13]). A pair $(\tilde{F}, A)$ is called a fuzzy soft set over $\mathrm{U}$, where $\tilde{F}$ is a mapping given by $\tilde{F}: A \rightarrow I^{U}$.

Definition 2.3(see [3]). The pair (U, E) is called a soft universe. $F: E \rightarrow I^{U}, \mu: E \rightarrow I^{U}$, Let $F_{\mu}: E \rightarrow$ $I^{U} \times I^{U}$ be a function defined as follows: $\mathrm{F} \mu(\mathrm{e})=(\mathrm{F}(\mathrm{e})(\mathrm{h}), \mu(\mathrm{e})(\mathrm{h})), \forall h \in U$. Then $\mathrm{F} \mu$ is called a possibility fuzzy soft set (PFSS) over the soft universe (U, E). For each parameter $e \in E, F \mu(\mathrm{e})=(\mathrm{F}(\mathrm{e})(\mathrm{h}), \mu(\mathrm{e})(\mathrm{h}))$ indicates not only the degree of belongingness of the elements of $U$ in $F(e)$, but also the degree of possibility of such belongingness of the elements of $\mathrm{U}$ in $\mathrm{F}(\mathrm{e})$, which is represented by $\mu(\mathrm{e})$.

Definition 2.4(see [3]). Let $\mathrm{F} \mu$ and $G_{\delta}$ be two PFSSs over (U, E). F $\mu$ is said to be a possibility fuzzy soft subset of $G_{\delta}$, and writes $\mathrm{F} \mu \subseteq G_{\delta}$, if

$\mu(\mathrm{e})$ is a fuzzy subset of $\delta(\mathrm{e})$, for all $e \in E$,

$\mathrm{F}(\mathrm{e})$ is a fuzzy subset of $\mathrm{G}(\mathrm{e})$, for all $e \in E$.

Definition 2.5(see [3]). A PFSS is said to be a possibility null fuzzy soft set, denoted by $\varphi_{0}$, if $\varphi_{0}: E \rightarrow I^{U} \times I^{U}$ such that $\varphi_{0}(e)=(\mathrm{F}(\mathrm{e})(\mathrm{h}), \mu(\mathrm{e})(\mathrm{h}))$, where $\mathrm{F}(\mathrm{e})(\mathrm{h})=0$, and $\mu(\mathrm{e})(\mathrm{h})=0$, for all $e \in E, h \in U$.

Definition 2.6(see [3]). A PFSS is said to be a possibility absolute fuzzy soft set, denoted by A1, if $A_{1}: E \rightarrow I^{U} \times I^{U}$ such that $\mathrm{A} 1(\mathrm{e})=(\mathrm{F}(\mathrm{e})(\mathrm{h}), \mu(\mathrm{e})(\mathrm{h}))$, where $\mathrm{F}(\mathrm{e})(\mathrm{h})=1$ and $\mu(\mathrm{e})(\mathrm{h})=1$, for all $e \in E, h \in U$.

Definition 2.7(see [3]). Union of two PFSSs F $\mu$ and $G_{\delta}$, denoted by $F_{\mu} \tilde{\cup} G_{\delta}$, is a PFSS $H_{v}: E \rightarrow I^{U} \times I^{U}$ defined by $H_{v}(e)=(\mathrm{H}(\mathrm{e})(\mathrm{h}), v(\mathrm{e})(\mathrm{h})), \forall e \in E, h \in U$, such that $\mathrm{H}(\mathrm{e})=\mathrm{s}(\mathrm{F}(\mathrm{e}), \mathrm{G}(\mathrm{e}))$ and $v(\mathrm{e})=\mathrm{s}(\mu(\mathrm{e}), \delta(\mathrm{e}))$, where $\mathrm{s}$ is an s-norm.

Definition 2.8(see [3]). Intersection of two PFSSs F $\mu$ and $G_{\delta}$, denoted by $F_{\mu} \tilde{\cap} G_{\delta}$, is a PFSS $H_{v}: E \rightarrow I^{U} \times I^{U}$ defined by $H_{v}(e)=(\mathrm{H}(\mathrm{e})(\mathrm{h}), v(\mathrm{e})(\mathrm{h})), \forall e \in E, h \in U$, such that $\mathrm{H}(\mathrm{e})=\mathrm{t}(\mathrm{F}(\mathrm{e}), \mathrm{G}(\mathrm{e}))$ and $v(\mathrm{e})=\mathrm{t}(\mu(\mathrm{e}), \delta(\mathrm{e}))$, where $\mathrm{t}$ is an $\mathrm{t}$-norm.

Definition 2.9(see [17]). A triplet (F, G, A) is called a bipolar soft set over $\mathrm{U}$, where $\mathrm{F}$ and $\mathrm{G}$ are mappings, given by $F: A \rightarrow P(U)$ and $G: \neg A \rightarrow P(U)$ such that $F(e) \cap G(\neg e)=\Phi$ (Empty Set) for all $e \in A$.

In other words, a bipolar soft set over $\mathrm{U}$ gives two parameterized families of subsets of the universe $\mathrm{U}$ and the condition $F(e) \cap G(\neg e)=\Phi$, for all $e \in A$, is imposed as a consistency constraint. For each $e \in A, \mathrm{~F}$ (e) and $G(\neg e)$ are regarded as the set of e-approximate elements of the bipolar soft set (F, G, A). It is also observed that the relationship between the complement function and the defining function of a soft set is a particular case for the defining functions of a bipolar soft set, i.e. $\left(F, F^{C}, A\right)$ is a bipolar soft set over U. The difference occurs due to the presence of uncertainty or hesitation or the lack of knowledge in defining the membership function. We name this uncertainty or gray area as the approximation for the degree of hesitation. Thus the union of three approximations i.e. e-approximation, $\neg e$-approximation, and approximation of hesitation is $\mathrm{U}$. We note that $\Phi \subseteq\{U-F(e) \cup G(\neg e)\} \subseteq$ 
$\mathrm{U}$, for each $e \in A$. So, we may approximate the degree of hesitation in (F, G, A) by an allied soft set (H, A) defined over $\mathrm{U}$, where $\mathrm{H}(\mathrm{e})=\mathrm{U}-\{F(e) \cup G(\neg e)\}$, for all $e \in A$.

Definition 2.10(see [17]). The complement of a bipolar soft set (F, G, A) is denoted by $(\mathrm{F}, \mathrm{G}, \mathrm{A}) \mathrm{c}$ and is defined by $(\mathrm{F}, \mathrm{G}, \mathrm{A}) \mathrm{c}=(\mathrm{Fc}, \mathrm{Go}, \mathrm{A})$, where $\mathrm{Fc}$ and $\mathrm{Go}$ are mappings given by $\mathrm{Fc}(\mathrm{e})=\mathrm{G}(\neg e)$ and $\mathrm{Go}(\neg e)=\mathrm{F}(\mathrm{e})$, for all $e \in A$.

\section{BIPOLAR POSSIBILITY FUZZY SOFT SETS}

Definition 3.1. A triplet $\left(\mathrm{F} \mu, G_{\delta}, \mathrm{A}\right)$ is called a bipolar possibility fuzzy soft set over $\mathrm{U}$, where $\mathrm{F} \mu$ and $G_{\delta}$ are mappings, given by $F_{\mu}: A \rightarrow \operatorname{PFSS}(U)$ and $G_{\delta}: \neg A \rightarrow \operatorname{PFSS}(U)$, let $\mathrm{F} \mu$ and $G_{\delta}$ be a function defined as: $\mathrm{F} \mu(\mathrm{e})=$ $(\mathrm{F}(\mathrm{e})(\mathrm{h}), \mu(\mathrm{e})(\mathrm{h})), G_{\delta}(\neg e)=(G(\neg e)(h), \delta(\neg e)(h)), \forall h \in U, e \in E$, where $\mathrm{F}(\mathrm{e})(\mathrm{h})$ indicates the degree of eapproximation belongingness of $\mathrm{h}, G(\neg e)(h)$ indicates the degree of $\neg e$-approximation belongingness of $\mathrm{h}$, and $\mathrm{F}$ $(\mathrm{e})(\mathrm{h})+G(\neg e)(h)$ should be in $[0,1]$.

Example 3.2. Let $\mathrm{U}=\{\mathrm{m} 1, \mathrm{~m} 2, \mathrm{~m} 3, \mathrm{~m} 4\}$ be the set of four men under consideration and $\mathrm{E}=\{\mathrm{e} 1=$ Educated, e2 $=$ Government employee, e $3=$ Businessman, e $4=$ Smart $\}$ be the set of parameters and $\mathrm{A}=\{\mathrm{e} 1, \mathrm{e} 2\} \subseteq \mathrm{E}$, then suppose the bipolar possibility fuzzy soft set $\left(\mathrm{F} \mu, G_{\delta} \mathrm{A}\right)$ is given as follows:

$$
\begin{aligned}
& F_{\mu}\left(e_{1}\right)=\left\{\left(\frac{m_{1}}{0.2}, 0.5\right),\left(\frac{m_{2}}{0.3}, 0.6\right),\left(\frac{m_{3}}{0.6}, 0.4\right),\left(\frac{m_{4}}{0.5}, 0.7\right)\right\}, \quad G_{\delta}\left(\neg e_{1}\right)=\left\{\left(\frac{m_{1}}{0.4}, 0.5\right),\left(\frac{m_{2}}{0.5}, 0.8\right),\left(\frac{m_{3}}{0.3}, 0.9\right),\left(\frac{m_{4}}{0.5}, 0.8\right)\right\}, \\
& F_{\mu}\left(e_{2}\right)=\left\{\left(\frac{m_{1}}{0.6}, 0.4\right),\left(\frac{m_{2}}{0.7}, 0.6\right),\left(\frac{m_{3}}{0.5}, 0.7\right),\left(\frac{m_{4}}{0.6}, 0.3\right)\right\}, \quad G_{\delta}\left(\neg e_{2}\right)=\left\{\left(\frac{m_{1}}{0.2}, 0.6\right),\left(\frac{m_{2}}{0.2}, 0.8\right),\left(\frac{m_{3}}{0.4}, 0.8\right),\left(\frac{m_{4}}{0.3}, 0.5\right)\right\} .
\end{aligned}
$$

Definition 3.3. For two bipolar possibility fuzzy soft sets $\left(\mathrm{F} \mu, G_{\delta}, \mathrm{A}\right)$ and $(\mathrm{R} \alpha, \mathrm{S} \beta, \mathrm{B})$ over a common universe $\mathrm{U}$, then $\left(\mathrm{F} \mu, G_{\delta}, \mathrm{A}\right)$ is a bipolar possibility fuzzy soft subset of $(\mathrm{R} \alpha, \mathrm{S} \beta, \mathrm{B})$, if

(1) $\mathrm{A} \subseteq \mathrm{B}$,

(2) $\mathrm{F} \mu(\mathrm{e}) \subseteq \mathrm{R} \alpha(\mathrm{e})$ and $G_{\delta}(\neg e) \supseteq \mathrm{S} \beta(\neg e)$ for all $e \in A$.

This relationship is denoted by $\left(F_{\mu}, G_{\delta}, A\right) \tilde{\subseteq}\left(R_{\alpha}, S_{\beta}, B\right)$. Similarly $\left(\mathrm{F} \mu, G_{\delta}, \mathrm{A}\right)$ is said to be a bipolar possibility fuzzy soft superset of $(\mathrm{R} \alpha, \mathrm{S} \beta, \mathrm{B})$, if $(\mathrm{R} \alpha, \mathrm{S} \beta, \mathrm{B})$ is a bipolar possibility fuzzy soft subset of $\left(\mathrm{F} \mu, G_{\delta}, \mathrm{A}\right)$, denoted by $\left(F_{\mu}, G_{\delta}, A\right) \supseteq\left(R_{\alpha}, S_{\beta}, B\right)$.

Example 3.4. Consider Example 3.2, let $\mathrm{B}=\{\mathrm{e} 1, \mathrm{e} 2, \mathrm{e} 3\} \subseteq \mathrm{E},(\mathrm{R} \alpha, \mathrm{S} \beta$, and $\mathrm{B})$ is given as follows:

$$
\begin{aligned}
& R_{\alpha}\left(e_{1}\right)=\left\{\left(\frac{m_{1}}{0.5}, 0.7\right),\left(\frac{m_{2}}{0.4}, 0.7\right),\left(\frac{m_{3}}{0.7}, 0.4\right),\left(\frac{m_{4}}{0.5}, 0.8\right)\right\}, S_{\beta}\left(-e_{1}\right)=\left\{\left(\frac{m_{1}}{0.3}, 0.2\right),\left(\frac{m_{2}}{0.4}, 0.7\right),\left(\frac{m_{3}}{0.3}, 0.2\right),\left(\frac{m_{4}}{0.3}, 0.6\right)\right\}, \\
& R_{\alpha}\left(e_{2}\right)=\left\{\left(\frac{m_{1}}{0.7}, 0.6\right),\left(\frac{m_{2}}{0.8}, 0.7\right),\left(\frac{m_{3}}{0.6}, 0.8\right),\left(\frac{m_{4}}{0.7}, 0.5\right)\right\}, S_{\beta}\left(-e_{2}\right)=\left\{\left(\frac{m_{1}}{0.1}, 0.3\right),\left(\frac{m_{2}}{0.2}, 0.6\right),\left(\frac{m_{3}}{0.3}, 0.5\right),\left(\frac{m_{4}}{0.2}, 0.3\right)\right\}, \\
& R_{\alpha}\left(e_{3}\right)=\left\{\left(\frac{m_{1}}{0.7}, 0.5\right),\left(\frac{m_{2}}{0.8}, 0.4\right),\left(\frac{m_{3}}{0.5}, 0.6\right),\left(\frac{m_{4}}{0.3}, 0.5\right)\right\}, S_{\beta}\left(-e_{3}\right)=\left\{\left(\frac{m_{1}}{0.8}, 0.6\right),\left(\frac{m_{2}}{0.4}, 0.6\right),\left(\frac{m_{3}}{0.1}, 0.5\right),\left(\frac{m_{4}}{0.3}, 0.7\right)\right\} .
\end{aligned}
$$

$\operatorname{Then}\left(F_{\mu}, G_{\delta}, A\right) \tilde{\subseteq}\left(R_{\alpha}, S_{\beta}, B\right)$.

Definition 3.5. Two bipolar possibility fuzzy soft sets $\left(\mathrm{F} \mu, G_{\delta} \mathrm{A}\right)$ and $(\mathrm{R} \alpha, \mathrm{S} \beta, \mathrm{B})$ over a common universe $\mathrm{U}$ are said to be equal if $\left(\mathrm{F} \mu, G_{\delta} \mathrm{A}\right)$ is a bipolar possibility fuzzy soft subset of $(\mathrm{R} \alpha, \mathrm{S} \beta, \mathrm{B})$ and $(\mathrm{R} \alpha, \mathrm{S} \beta, \mathrm{B})$ is a bipolar possibility fuzzy soft subset of $\left(\mathrm{F} \mu, G_{\delta} \mathrm{A}\right)$.

Definition 3.6. The complement of a bipolar possibility fuzzy soft set $\left(\mathrm{F} \mu, G_{\delta} \mathrm{A}\right)$ is denoted by $\left(F_{\mu}, G_{\delta}, A\right)^{c}$ and is defined by $\left(F_{\mu}, G_{\delta}, A\right)^{c}=\left(F_{\mu}^{c}, G_{\delta}{ }^{c}, A\right)$, where $F_{\mu}{ }^{c}$ and $G_{\delta}{ }^{c}$ are mappings given by $F_{\mu}{ }^{c}(e)=G_{\delta}(\neg e)$ and $G_{\delta}{ }^{c}(\neg e)=F_{\mu}(e)$, for all $e \in A$.

Example 3.7. Reconsider Example 3.2, the complement of the bipolar possibility fuzzy soft set $\left(\mathrm{F} \mu, G_{\delta} \mathrm{A}\right)$ is 


$$
\begin{gathered}
F_{\mu}\left(e_{1}\right)^{c}=\left\{\left(\frac{m_{1}}{0.4}, 0.5\right),\left(\frac{m_{2}}{0.5}, 0.8\right),\left(\frac{m_{3}}{0.3}, 0.9\right),\left(\frac{m_{4}}{0.5}, 0.8\right)\right\}, \quad G_{\delta}\left(\neg e_{1}\right)^{c}=\left\{\left(\frac{m_{1}}{0.2}, 0.5\right),\left(\frac{m_{2}}{0.3}, 0.6\right),\left(\frac{m_{3}}{0.6}, 0.4\right),\left(\frac{m_{4}}{0.5}, 0.7\right)\right\}, \\
F_{\mu}\left(e_{2}\right)^{c}=\left\{\left(\frac{m_{1}}{0.2}, 0.6\right),\left(\frac{m_{2}}{0.2}, 0.8\right),\left(\frac{m_{3}}{0.4}, 0.8\right),\left(\frac{m_{4}}{0.3}, 0.5\right)\right\}, \quad G_{\delta}\left(\neg e_{2}\right)^{c}=\left\{\left(\frac{m_{1}}{0.6}, 0.4\right),\left(\frac{m_{2}}{0.7}, 0.6\right),\left(\frac{m_{3}}{0.5}, 0.7\right),\left(\frac{m_{4}}{0.6}, 0.3\right)\right\} .
\end{gathered}
$$

Definition 3.8. A bipolar possibility fuzzy soft set over $U$ is said to be a relative null bipolar possibility fuzzy soft set, denoted by $(\Phi, \Delta, \mathrm{A})$, if for all $e \in A, \Phi(\mathrm{e})=\varphi_{0}(e)$ and $\Delta(\neg e)=\mathrm{A} 1(\neg e)$, for all $e \in A$.

Definition 3.9. A bipolar possibility fuzzy soft set over $U$ is said to be a relative absolute bipolar possibility fuzzy soft set, denoted by $(\Delta, \Phi, \mathrm{A})$, if for all $e \in A, \Delta(\mathrm{e})=\mathrm{A} 1(\mathrm{e})$ and $\Phi(\neg e)=\varphi_{0}(\neg e)$, for all $e \in A$.

Definition 3.10. Extended union of two bipolar possibility fuzzy soft sets $\left(\mathrm{F} \mu, G_{\delta}, \mathrm{A}\right)$ and $(\mathrm{R} \alpha, \mathrm{S} \beta, \mathrm{B})$ over a common universe $\mathrm{U}$ is the bipolar possibility fuzzy soft set $\left(H_{\omega}, I_{v}, C\right)$ over $\mathrm{U}$, where $C=A \cup B$ and for all $e \in C$,

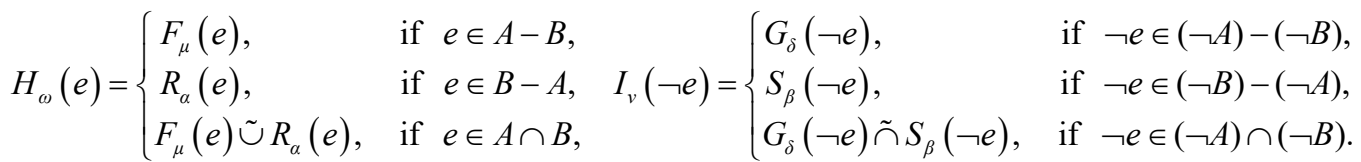

It is denoted by $\left(F_{\mu}, G_{\delta}, A\right) \bigcup_{E}\left(R_{\alpha}, S_{\beta}, B\right)=\left(H_{\omega}, I_{v}, C\right)$.

Definition 3.11. Extended intersection of two bipolar possibility fuzzy soft sets $\left(\mathrm{F} \mu, G_{\delta} \mathrm{A}\right)$ and $(\mathrm{R} \alpha, \mathrm{S} \beta, \mathrm{B})$ over a common universe $\mathrm{U}$ is the bipolar possibility fuzzy soft set $\left(H_{\omega}, I_{v}, C\right)$ over $\mathrm{U}$, where $C=A \cup B$ and for all $e \in C$,

$$
H_{\omega}(e)= \begin{cases}F_{\mu}(e), & \text { if } e \in A-B, \\
R_{\alpha}(e), & \text { if } e \in B-A, \quad I_{v}(\neg e)=\left\{\begin{array}{ll}
G_{\delta}(\neg e), & \text { if } \neg e \in(\neg A)-(\neg B), \\
F_{\mu}(e) \tilde{\cap} R_{\alpha}(e), & \text { if } e \in A \cap B),
\end{array} \quad \text { if } \neg e \in(\neg B)-(\neg A),\right. \\
G_{\delta}(\neg e) \tilde{\cup} S_{\beta}(\neg e), & \text { if } \neg e \in(\neg A) \cap(\neg B) .\end{cases}
$$

It is denoted by $\left(F_{\mu}, G_{\delta}, A\right) \bigcap_{E}\left(R_{\alpha}, S_{\beta}, B\right)=\left(H_{\omega}, I_{v}, C\right)$.

Definition 3.12. Restricted union of two bipolar possibility fuzzy soft sets $\left(\mathrm{F} \mu, G_{\delta} \mathrm{A}\right)$ and $(\mathrm{R} \alpha, \mathrm{S} \beta, \mathrm{B})$ over a common universe $\mathrm{U}$ is the bipolar possibility fuzzy soft set $\left(H_{\omega}, I_{v}, C\right)$ over $\mathrm{U}$, where $C=A \cap B$ is non-empty and for all $e \in C, H_{\omega}(e)=F_{\mu}(e) \tilde{U} R_{\alpha}(e)$ and $I_{\nu}(\neg e)=G_{\delta}(\neg e) \tilde{\cap} S_{\beta}(\neg e)$. It is denoted by $\left(F_{\mu}, G_{\delta}, A\right) \bigcup_{R}\left(R_{\alpha}, S_{\beta}, B\right)=$ $\left(H_{\omega}, I_{v}, C\right)$.

Definition 3.13. Restricted intersection of two bipolar possibility fuzzy soft sets $\left(\mathrm{F} \mu, G_{\delta} \mathrm{A}\right)$ and $(\mathrm{R} \alpha, \mathrm{S} \beta, \mathrm{B})$ over a common universe $\mathrm{U}$ is the bipolar possibility fuzzy soft $\operatorname{set}\left(H_{\omega}, I_{v}, C\right)$, where $C=A \cap B$ is non-empty and for all $e \in C, H_{\omega}(e)=F_{\mu}(e) \tilde{\cap} R_{\alpha}(e)$ and $I_{v}(\neg e)=G_{\delta}(\neg e) \tilde{\cup} S_{\beta}(\neg e)$. It is denoted by $\left(F_{\mu}, G_{\delta}, A\right) \bigcap_{R}\left(R_{\alpha}, S_{\beta}, B\right)=$ $\left(H_{\omega}, I_{v}, C\right)$.

Example 3.14. Let $U=\{h 1, h 2, h 3\}$ be the set of three houses under consideration and $E=\{e 1=$ wooden, e2 =cheap, e $3=$ in good repair, e $4=$ traditional $\}$ be the set of parameters, then $\neg E=\left\{\neg e_{1}=\right.$ marbled, $\neg e_{2}=$ expensive, $\neg e_{3}=$ in bad repair, $\neg e_{4}=$ modern $\}$. Suppose that $\mathrm{A}=\{\mathrm{e} 1, \mathrm{e} 2, \mathrm{e} 3\}$, and $\mathrm{B}=\{\mathrm{e} 1, \mathrm{e} 2, \mathrm{e} 4\}$. The bipolar possibility fuzzy soft sets $\left(\mathrm{F} \mu, G_{\delta}, \mathrm{A}\right)$ and $(\mathrm{R} \alpha, \mathrm{S} \beta, \mathrm{B})$ describe the "requirements of the houses" which $\mathrm{Mr}$. X and Mr. Y are going to buy respectively. Suppose that

$$
\begin{aligned}
& F_{\mu}\left(e_{1}\right)=\left\{\left(\frac{h_{1}}{0.2}, 0.5\right),\left(\frac{h_{2}}{0.3}, 0.6\right),\left(\frac{h_{3}}{0.6}, 0.4\right)\right\}, \quad G_{\delta}\left(\neg e_{1}\right)=\left\{\left(\frac{h_{1}}{0.4}, 0.5\right),\left(\frac{h_{2}}{0.5}, 0.8\right),\left(\frac{h_{3}}{0.3}, 0.9\right)\right\}, \\
& F_{\mu}\left(e_{2}\right)=\left\{\left(\frac{h_{1}}{0.6}, 0.4\right),\left(\frac{h_{2}}{0.7}, 0.7\right),\left(\frac{h_{3}}{0.5}, 0.7\right)\right\}, \quad G_{\delta}\left(\neg e_{2}\right)=\left\{\left(\frac{h_{1}}{0.2}, 0.6\right),\left(\frac{h_{2}}{0.2}, 0.4\right),\left(\frac{h_{3}}{0.4}, 0.8\right)\right\}, \\
& F_{\mu}\left(e_{3}\right)=\left\{\left(\frac{h_{1}}{0.5}, 0.3\right),\left(\frac{h_{2}}{0.4}, 0.3\right),\left(\frac{h_{3}}{0.5}, 0.6\right)\right\}, \quad G_{\delta}\left(\neg e_{3}\right)=\left\{\left(\frac{h_{1}}{0.3}, 0.8\right),\left(\frac{h_{2}}{0.6}, 0.5\right),\left(\frac{h_{3}}{0.2}, 0.5\right)\right\} .
\end{aligned}
$$

And 


$$
\begin{array}{ll}
R_{\alpha}\left(e_{1}\right)=\left\{\left(\frac{h_{1}}{0.3}, 0.6\right),\left(\frac{h_{2}}{0.5}, 0.3\right),\left(\frac{h_{3}}{0.4}, 0.3\right)\right\}, & S_{\beta}\left(\neg e_{1}\right)=\left\{\left(\frac{h_{1}}{0.5}, 0.4\right),\left(\frac{h_{2}}{0.4}, 0.8\right),\left(\frac{h_{3}}{0.6}, 0.3\right)\right\}, \\
R_{\alpha}\left(e_{2}\right)=\left\{\left(\frac{h_{1}}{0.4}, 0.8\right),\left(\frac{h_{2}}{0.6}, 0.7\right),\left(\frac{h_{3}}{0.2}, 0.5\right)\right\}, & S_{\beta}\left(\neg e_{2}\right)=\left\{\left(\frac{h_{1}}{0.3}, 0.8\right),\left(\frac{h_{2}}{0.4}, 0.7\right),\left(\frac{h_{3}}{0.7}, 0.8\right)\right\}, \\
R_{\alpha}\left(e_{4}\right)=\left\{\left(\frac{h_{1}}{0.6}, 0.3\right),\left(\frac{h_{2}}{0.3}, 0.6\right),\left(\frac{h_{3}}{0.7}, 0.8\right)\right\}, & S_{\beta}\left(\neg e_{4}\right)=\left\{\left(\frac{h_{1}}{0.3}, 0.6\right),\left(\frac{h_{2}}{0.4}, 0.5\right),\left(\frac{h_{3}}{0.2}, 0.6\right)\right\} .
\end{array}
$$

$\operatorname{Let}\left(F_{\mu}, G_{\delta}, A\right) \bigcup_{E}\left(R_{\alpha}, S_{\beta}, B\right)=\left(H_{\omega}^{1}, I_{v}^{1}, A \cup B\right)$, then

$$
\begin{array}{ll}
H_{\omega}^{1}\left(e_{1}\right)=\left\{\left(\frac{h_{1}}{0.3}, 0.6\right),\left(\frac{h_{2}}{0.5}, 0.6\right),\left(\frac{h_{3}}{0.6}, 0.4\right)\right\}, & I_{v}^{1}\left(\neg e_{1}\right)=\left\{\left(\frac{h_{1}}{0.4}, 0.4\right),\left(\frac{h_{2}}{0.4}, 0.8\right),\left(\frac{h_{3}}{0.3}, 0.3\right)\right\}, \\
H_{\omega}^{1}\left(e_{2}\right)=\left\{\left(\frac{h_{1}}{0.6}, 0.8\right),\left(\frac{h_{2}}{0.7}, 0.7\right),\left(\frac{h_{3}}{0.5}, 0.7\right)\right\}, & I_{v}^{1}\left(\neg e_{2}\right)=\left\{\left(\frac{h_{1}}{0.2}, 0.6\right),\left(\frac{h_{2}}{0.2}, 0.4\right),\left(\frac{h_{3}}{0.4}, 0.8\right)\right\}, \\
H_{\omega}^{1}\left(e_{3}\right)=\left\{\left(\frac{h_{1}}{0.5}, 0.3\right),\left(\frac{h_{2}}{0.4}, 0.3\right),\left(\frac{h_{3}}{0.5}, 0.6\right)\right\}, & I_{v}^{1}\left(\neg e_{3}\right)=\left\{\left(\frac{h_{1}}{0.3}, 0.8\right),\left(\frac{h_{2}}{0.6}, 0.5\right),\left(\frac{h_{3}}{0.2}, 0.5\right)\right\}, \\
H_{\omega}^{1}\left(e_{4}\right)=\left\{\left(\frac{h_{1}}{0.6}, 0.3\right),\left(\frac{h_{2}}{0.3}, 0.6\right),\left(\frac{h_{3}}{0.7}, 0.8\right)\right\}, & I_{v}^{1}\left(\neg e_{4}\right)=\left\{\left(\frac{h_{1}}{0.3}, 0.6\right),\left(\frac{h_{2}}{0.4}, 0.5\right),\left(\frac{h_{3}}{0.2}, 0.6\right)\right\} .
\end{array}
$$

$\operatorname{Let}\left(F_{\mu}, G_{\delta}, A\right) \bigcap_{E}\left(R_{\alpha}, S_{\beta}, B\right)=\left(H_{\omega}^{2}, I_{v}^{2}, A \cup B\right)$, then

$$
\begin{array}{ll}
H_{\omega}^{2}\left(e_{1}\right)=\left\{\left(\frac{h_{1}}{0.2}, 0.5\right),\left(\frac{h_{2}}{0.3}, 0.3\right),\left(\frac{h_{3}}{0.4}, 0.3\right)\right\}, & I_{v}^{2}\left(\neg e_{1}\right)=\left\{\left(\frac{h_{1}}{0.5}, 0.5\right),\left(\frac{h_{2}}{0.5}, 0.8\right),\left(\frac{h_{3}}{0.6}, 0.9\right)\right\}, \\
H_{\omega}^{2}\left(e_{2}\right)=\left\{\left(\frac{h_{1}}{0.4}, 0.4\right),\left(\frac{h_{2}}{0.6}, 0.7\right),\left(\frac{h_{3}}{0.2}, 0.5\right)\right\}, & I_{v}^{2}\left(\neg e_{2}\right)=\left\{\left(\frac{h_{1}}{0.3}, 0.8\right),\left(\frac{h_{2}}{0.4}, 0.7\right),\left(\frac{h_{3}}{0.7}, 0.8\right)\right\}, \\
H_{\omega}^{2}\left(e_{3}\right)=\left\{\left(\frac{h_{1}}{0.5}, 0.3\right),\left(\frac{h_{2}}{0.4}, 0.3\right),\left(\frac{h_{3}}{0.5}, 0.6\right)\right\}, & I_{v}^{2}\left(\neg e_{3}\right)=\left\{\left(\frac{h_{1}}{0.3}, 0.8\right),\left(\frac{h_{2}}{0.6}, 0.5\right),\left(\frac{h_{3}}{0.2}, 0.5\right)\right\}, \\
H_{\omega}^{2}\left(e_{4}\right)=\left\{\left(\frac{h_{1}}{0.6}, 0.3\right),\left(\frac{h_{2}}{0.3}, 0.6\right),\left(\frac{h_{3}}{0.7}, 0.8\right)\right\}, & I_{v}^{2}\left(\neg e_{4}\right)=\left\{\left(\frac{h_{1}}{0.3}, 0.6\right),\left(\frac{h_{2}}{0.4}, 0.5\right),\left(\frac{h_{3}}{0.2}, 0.6\right)\right\} .
\end{array}
$$

Let $\left(F_{\mu}, G_{\delta}, A\right) \bigcup_{R}\left(R_{\alpha}, S_{\beta}, B\right)=\left(H_{\omega}^{3}, I_{v}^{3}, A \cap B\right)$, then

$$
\begin{aligned}
& H_{\omega}^{3}\left(e_{1}\right)=\left\{\left(\frac{h_{1}}{0.3}, 0.6\right),\left(\frac{h_{2}}{0.5}, 0.6\right),\left(\frac{h_{3}}{0.6}, 0.4\right)\right\}, \quad I_{v}^{3}\left(\neg e_{1}\right)=\left\{\left(\frac{h_{1}}{0.4}, 0.4\right),\left(\frac{h_{2}}{0.4}, 0.8\right),\left(\frac{h_{3}}{0.3}, 0.3\right)\right\}, \\
& H_{\omega}^{3}\left(e_{2}\right)=\left\{\left(\frac{h_{1}}{0.6}, 0.8\right),\left(\frac{h_{2}}{0.7}, 0.7\right),\left(\frac{h_{3}}{0.5}, 0.7\right)\right\}, \quad I_{v}^{3}\left(\neg e_{2}\right)=\left\{\left(\frac{h_{1}}{0.2}, 0.6\right),\left(\frac{h_{2}}{0.2}, 0.4\right),\left(\frac{h_{3}}{0.4}, 0.8\right)\right\} .
\end{aligned}
$$

Let $\left(F_{\mu}, G_{\delta}, A\right) \bigcap_{R}\left(R_{\alpha}, S_{\beta}, B\right)=\left(H_{\omega}^{4}, I_{v}^{4}, A \cap B\right)$, then

$$
\begin{array}{ll}
H_{\omega}^{4}\left(e_{1}\right)=\left\{\left(\frac{h_{1}}{0.2}, 0.5\right),\left(\frac{h_{2}}{0.3}, 0.3\right),\left(\frac{h_{3}}{0.4}, 0.3\right)\right\}, & I_{v}^{4}\left(\neg e_{1}\right)=\left\{\left(\frac{h_{1}}{0.5}, 0.5\right),\left(\frac{h_{2}}{0.5}, 0.8\right),\left(\frac{h_{3}}{0.6}, 0.9\right)\right\}, \\
H_{\omega}^{4}\left(e_{2}\right)=\left\{\left(\frac{h_{1}}{0.4}, 0.4\right),\left(\frac{h_{2}}{0.6}, 0.7\right),\left(\frac{h_{3}}{0.2}, 0.5\right)\right\}, & I_{v}^{4}\left(\neg e_{2}\right)=\left\{\left(\frac{h_{1}}{0.3}, 0.8\right),\left(\frac{h_{2}}{0.4}, 0.7\right),\left(\frac{h_{3}}{0.7}, 0.8\right)\right\} .
\end{array}
$$

Proposition 3.15. Let $\left(\mathrm{F} \mu, G_{\delta}, \mathrm{A}\right)$ and $(\mathrm{R} \alpha, \mathrm{S} \beta$, A) be bipolar possibility fuzzy soft sets over $\mathrm{U}$, then 
(1) $\left(F_{\mu}, G_{\delta}, A\right) \bigcup_{E}\left(R_{\alpha}, S_{\beta}, A\right)=\left(F_{\mu}, G_{\delta}, A\right) \bigcup_{R}\left(R_{\alpha}, S_{\beta}, A\right)$,

(2) $\left(F_{\mu}, G_{\delta}, A\right) \bigcap_{E}\left(R_{\alpha}, S_{\beta}, A\right)=\left(F_{\mu}, G_{\delta}, A\right) \bigcap_{R}\left(R_{\alpha}, S_{\beta}, A\right)$,

(3) $\left(F_{\mu}, G_{\delta}, A\right) \bigcup_{E}\left(F_{\mu}, G_{\delta}, A\right)=\left(F_{\mu}, G_{\delta}, A\right) ;\left(F_{\mu}, G_{\delta}, A\right) \bigcap_{E}\left(F_{\mu}, G_{\delta}, A\right)=\left(F_{\mu}, G_{\delta}, A\right)$,

(4) $\left(F_{\mu}, G_{\delta}, A\right) \bigcup_{R}\left(F_{\mu}, G_{\delta}, A\right)=\left(F_{\mu}, G_{\delta}, A\right) ; \quad\left(F_{\mu}, G_{\delta}, A\right) \bigcap_{R}\left(F_{\mu}, G_{\delta}, A\right)=\left(F_{\mu}, G_{\delta}, A\right)$,

(5) $\left(F_{\mu}, G_{\delta}, A\right) \bigcup_{E}(\Phi, \Delta, A)=\left(F_{\mu}, G_{\delta}, A\right) ; \quad\left(F_{\mu}, G_{\delta}, A\right) \bigcap_{E}(\Phi, \Delta, A)=(\Phi, \Delta, A)$,

(6) $\left(F_{\mu}, G_{\delta}, A\right) \bigcup_{R}(\Phi, \Delta, A)=\left(F_{\mu}, G_{\delta}, A\right) ; \quad\left(F_{\mu}, G_{\delta}, A\right) \bigcap_{R}(\Phi, \Delta, A)=(\Phi, \Delta, A)$,

(7) $\left(F_{\mu}, G_{\delta}, A\right) \bigcup_{E}(\Delta, \Phi, A)=(\Delta, \Phi, A) ; \quad\left(F_{\mu}, G_{\delta}, A\right) \bigcap_{E}(\Delta, \Phi, A)=\left(F_{\mu}, G_{\delta}, A\right)$,

(8) $\left(F_{\mu}, G_{\delta}, A\right) \bigcup_{R}(\Delta, \Phi, A)=(\Delta, \Phi, A) ; \quad\left(F_{\mu}, G_{\delta}, A\right) \bigcap_{R}(\Delta, \Phi, A)=\left(F_{\mu}, G_{\delta}, A\right)$.

Proof. Straightforward.

Proposition 3.16. Let $\left(\mathrm{F} \mu, G_{\delta} \mathrm{A}\right)$ and $(\mathrm{R} \alpha, \mathrm{S} \beta, \mathrm{B})$ be two bipolar possibility fuzzy soft sets over a common universe $\mathrm{U}$. Then the following are true:

(1) $\left(\left(F_{\mu}, G_{\delta}, A\right) \bigcup_{R}\left(R_{\alpha}, S_{\beta}, B\right)\right)^{c}=\left(F_{\mu}, G_{\delta}, A\right)^{c} \bigcap_{R}\left(R_{\alpha}, S_{\beta}, B\right)^{c}$,

(2) $\left(\left(F_{\mu}, G_{\delta}, A\right) \bigcap_{R}\left(R_{\alpha}, S_{\beta}, B\right)\right)^{c}=\left(F_{\mu}, G_{\delta}, A\right)^{c} \bigcup_{R}\left(R_{\alpha}, S_{\beta}, B\right)^{c}$,

(3) $\left(\left(F_{\mu}, G_{\delta}, A\right) \bigcup_{E}\left(R_{\alpha}, S_{\beta}, B\right)\right)^{c}=\left(F_{\mu}, G_{\delta}, A\right)^{c} \bigcap_{E}\left(R_{\alpha}, S_{\beta}, B\right)^{c}$,

(4) $\left(\left(F_{\mu}, G_{\delta}, A\right) \bigcap_{E}\left(R_{\alpha}, S_{\beta}, B\right)\right)^{c}=\left(F_{\mu}, G_{\delta}, A\right)^{c} \bigcup_{E}\left(R_{\alpha}, S_{\beta}, B\right)^{c}$.

Proof. Here we only prove the result (1), the others can be obtained similarity.

(1) Support that $\left(F_{\mu}, G_{\delta}, A\right) \bigcup_{R}\left(R_{\alpha}, S_{\beta}, B\right)=\left(H_{\omega}, I_{v}, C\right)$, where $C=A \cap B$, and for all $e \in C, h \in U, H_{\omega}(e)(h)$ $=(H(e)(h), \omega(e)(h))$, where $H(e)(h)=F(e)(h) \tilde{\cup} R(e)(h), \omega(e)(h)=\mu(e)(h) \tilde{\cup} \alpha(e)(h)$, and $I_{v}(\neg e)(h)=(I(\neg e)(h)$, $v(\neg e)(h))$, where $I_{v}(\neg e)(\mathrm{h})=G(\neg e)(h) \tilde{\cap} S(\neg e)(h), v(\neg e)(h)=\delta(\neg e)(h) \tilde{\cap} \beta(\neg e)(h)$.

So, we have $\left(\left(F_{\mu}, G_{\delta}, A\right) \bigcup_{R}\left(R_{\alpha}, S_{\beta}, B\right)\right)^{c}=\left(H_{\omega}, I_{v}, C\right)^{c}$, for all $e \in C, h \in U$,

$H_{\omega}^{c}(e)(h)=I_{v}(\neg e)(h)=(G(\neg e)(h) \tilde{\cap} S(\neg e)(h), \delta(\neg e)(h) \tilde{\cap} \beta(\neg e)(h))$,

$I_{v}^{c}(\neg \mathrm{e})(\mathrm{h})=H_{\omega}(e)(h)=(F(e)(h) \tilde{\cup} R(e)(h), \mu(e)(h) \tilde{\cup} \alpha(e)(h))$.

Assume that $\left(F_{\mu}, G_{\delta}, A\right)^{c} \bigcap_{R}\left(R_{\alpha}, S_{\beta}, B\right)^{c}=\left(K_{\gamma}, J_{\theta}, D\right)$, where $D=A \cap B$, and for all $e \in D, h \in U$

$K_{\gamma}(e)(h)=F_{\mu}^{c}(e)(h) \tilde{\cap} R_{\alpha}^{c}(e)(h)=(G(\neg e)(h) \tilde{\cap} S(\neg e)(h), \delta(\neg e)(h) \tilde{\cap} \beta(\neg e)(h))$,

$J_{\theta}(\neg e)(h)=G_{\delta}^{c}(\neg e)(h) \tilde{U} S_{\beta}^{c}(\neg e)(h)=(F(e)(h) \tilde{\cup} R(e)(h), \mu(e)(h) \tilde{\cup} \alpha(e)(h))$

Therefore, $\left(H_{\omega}, I_{v}, C\right)^{c}$ and $\left(K_{\gamma}, J_{\theta}, D\right)$ are the same bipolar possibility fuzzy soft set. Thus, $\left(\left(F_{\mu}, G_{\delta}, A\right) \bigcup_{R}\right.$ $\left.\left(R_{\alpha}, S_{\beta}, B\right)\right)^{c}=\left(F_{\mu}, G_{\delta}, A\right)^{c} \bigcap_{R}\left(R_{\alpha}, S_{\beta}, B\right)^{c}$.

Definition 3.17. If $\left(\mathrm{F} \mu, G_{\delta}, \mathrm{A}\right)$ and $(\mathrm{R} \alpha, \mathrm{S} \beta, \mathrm{B})$ are two bipolar possibility fuzzy soft sets over a common universe $\mathrm{U}$, then "(F $\left.\mu, G_{\delta}, \quad \mathrm{A}\right)$ AND $(\mathrm{R} \alpha, \quad \mathrm{S} \beta, \mathrm{B})$ ", denoted by $\left(F_{\mu}, G_{\delta}, A\right) \wedge\left(R_{\alpha}, S_{\beta}, B\right)$, is defined by $\left(F_{\mu}, G_{\delta}, A\right) \wedge\left(R_{\alpha}, S_{\beta}, B\right)=\left(H_{\omega}, I_{v}, A \times B\right)$, where, $H_{\omega}(a, b)=F_{\mu}(a) \tilde{\cap} R_{\alpha}(b)$, and $I_{\nu}(\neg a, \neg b)=G_{\delta}(\neg a) \tilde{\cup} S_{\beta}(\neg b)$, for all $(a, b) \in A \times B$.

Definition 3.18. If $\left(\mathrm{F} \mu, G_{\delta}, \mathrm{A}\right)$ and $(\mathrm{R} \alpha, \mathrm{S} \beta, \mathrm{B})$ are two bipolar possibility fuzzy soft sets over a common universe $\mathrm{U}$, then $\left(\mathrm{F} \mu, G_{\delta}, \quad \mathrm{A}\right) \quad \mathrm{OR} \quad(\mathrm{R} \alpha, \quad \mathrm{S} \beta, \quad \mathrm{B}) "$, denoted by $\left(F_{\mu}, G_{\delta}, A\right) \vee\left(R_{\alpha}, S_{\beta}, B\right)$, is defined by $\left(F_{\mu}, G_{\delta}, A\right) \vee\left(R_{\alpha}, S_{\beta}, B\right)=\left(H_{\omega}, I_{v}, A \times B\right)$, where $H_{\omega}(a, b)=F_{\mu}(a) \tilde{\cup} R_{\alpha}(b)$ and $I_{v}(\neg a, \neg b)=G_{\delta}(\neg a) \tilde{\cap} S_{\beta}(\neg b)$, for all $(a, b) \in A \times B$.

Proposition 3.19. If $\left(\mathrm{F} \mu, G_{\delta}, \mathrm{A}\right)$ and $(\mathrm{R} \alpha, \mathrm{S} \beta, \mathrm{B})$ are two bipolar possibility fuzzy soft sets over a common universe $\mathrm{U}$, then

(1) $\left(\left(F_{\mu}, G_{\delta}, A\right) \vee\left(R_{\alpha}, S_{\beta}, B\right)\right)^{c}=\left(F_{\mu}, G_{\delta}, A\right)^{c} \wedge\left(R_{\alpha}, S_{\beta}, B\right)^{c}$,

(2) $\left(\left(F_{\mu}, G_{\delta}, A\right) \wedge\left(R_{\alpha}, S_{\beta}, B\right)\right)^{c}=\left(F_{\mu}, G_{\delta}, A\right)^{c} \vee\left(R_{\alpha}, S_{\beta}, B\right)^{c}$. 
Proof. Straightforward.

\section{APPLICATION OF BIPOLAR POSSIBILITY FUZZY SOFT SETS IN A DECISION MAKING PROBLEM}

Suppose that $\left(\mathrm{F} \mu, G_{\delta} \mathrm{A}\right)$ is a bipolar possibility fuzzy soft set over $\mathrm{U}$. For each $h_{i} \in U, e_{k} \in A, b_{i}\left(e_{k}\right)$ and $d_{i}\left(\neg e_{k}\right)$ are defined as: $b_{i}\left(e_{k}\right)=F\left(e_{k}\right)\left(h_{i}\right) \times \mu\left(e_{k}\right)\left(h_{i}\right), d_{i}\left(\neg e_{k}\right)=G\left(\neg e_{k}\right)\left(h_{i}\right) \times \delta\left(\neg e_{k}\right)\left(h_{i}\right)$.

Definition 4.1. Let $\left(\mathrm{F} \mu, G_{\delta} \mathrm{A}\right)$ be a bipolar possibility fuzzy soft set over $\mathrm{U}, h_{i}, h_{j} \in U, e_{k} \in A$. The eapproximation membership value of hi exceeds or equals to the e-approximation membership value of $h_{j}$ with respect to the parameter $e_{k}$ represented by $b_{i}\left(e_{k}\right) \geq b_{j}\left(e_{k}\right)$. The corresponding characteristic function is defined as follows:

$$
C_{i j}\left(e_{k}\right)= \begin{cases}1, & \text { if } b_{i}\left(e_{k}\right) \geq b_{j}\left(e_{k}\right), \\ 0, & \text { otherwise. }\end{cases}
$$

While, the $\neg e$-approximation membership value of hi exceeds or equals to the $\neg e$-approximation membership value of $h_{j}$ with respect to the parameter $e_{k}$ represented by $d_{i}\left(\neg e_{k}\right) \geq d_{j}\left(\neg e_{k}\right)$. The corresponding characteristic function is defined as follows:

$$
C_{i j}\left(\neg e_{k}\right)=\left\{\begin{array}{cc}
-1, & \text { if } d_{i}\left(\neg e_{k}\right) \geq d_{j}\left(\neg e_{k}\right), \\
0, & \text { otherwise. }
\end{array}\right.
$$

Definition 4.2. (Comparison tables). Let $\left(\mathrm{F} \mu, G_{\delta} \mathrm{A}\right)$ be a bipolar possibility fuzzy soft set over $\mathrm{U}, \mathrm{A}=\{\mathrm{e} 1, \mathrm{e} 2$, e3, $\left.e_{k}\right), \neg A=\left\{\neg e_{1}, \neg e_{2}, \neg e_{3}, \ldots, \neg e_{k}\right\}$. The positive information comparison table is a square table in which the number of rows and the number of columns is equal and both are labeled by the object name of the universe such as $\mathrm{h} 1, \mathrm{~h} 2, \mathrm{~h} 3, h_{n}$ and the entries $h_{i j}$ defined as follows:

$$
h_{i j}=\sum_{l=1}^{k} C_{i j}\left(e_{l}\right) .
$$

While, the negative information comparison table is also a square table in which the number of rows and the number of columns are equal and both are labeled by the object name of the universe such as h1, h2, h3, $h_{n}$ and the entries $h_{i j}$ defined as follows:

$$
h_{i j}=\sum_{l=1}^{k} C_{i j}\left(\neg e_{l}\right) .
$$

Algorithm 4.3. The algorithm for the selection of the optimal choice is given as below:

(1) Input the set $\mathrm{A} \subseteq \mathrm{E}$ of choice of parameters and the bipolar possibility fuzzy soft $\operatorname{set}\left(\mathrm{F} \mu, G_{\delta}, \mathrm{A}\right)$.

(2) Compute the $b_{i}\left(e_{k}\right)$ aid $d_{i}\left(\neg e_{k}\right)$ of $\left(\mathrm{F} \mu, G_{\delta}, \mathrm{A}\right)$ in tabular forms, respectively.

(3) Compute the comparison tables of positive information and negative information.

(4) Compute positive information score and negative information score.

(5) Add positive information score and negative information score.

(6) Choose the optimal alternative $h_{k}$, which has the maximal score. If $\mathrm{k}$ has more than one values, then any one of $h_{k}$ can be chosen.

To illustrate the algorithm above, we consider the following example.

Example 4.4. Let $\mathrm{U}=\{\mathrm{c} 1, \mathrm{c} 2, \mathrm{c} 3, \mathrm{c} 4, \mathrm{c5}, \mathrm{c6}, \mathrm{c} 7, \mathrm{c} 8\}$ be the set of candidates who have applied for a job position of Office Representative in Customer Care Centre of a company. Let $\mathrm{E}=\{\mathrm{e} 1, \mathrm{e} 2, \mathrm{e} 3, \mathrm{e} 4, \mathrm{e} 5, \mathrm{e} 6, \mathrm{e} 7, \mathrm{e} 8\}=\{\mathrm{Hard}$ Working, Optimism, Enthusiasm, Individualism, Imaginative, Flexibility, Decisiveness, Self-confidence $\}$ and $\neg E=\left\{\neg e_{1}, \neg e_{2}, \neg e_{3}, \neg e_{4}, \neg e_{5}, \neg e_{6}, \neg e_{7}, \neg e_{8}\right\}=\{$ Negligent, Pessimism, Half-hearted, Dependence, Unimaginative, Rigidity, Indecisiveness, Shyness $\}$. Here the gray area is obviously the moderate form of parameters. Let the bipolar possibility fuzzy soft set $\left(\mathrm{F} \mu, G_{\delta}, \mathrm{E}\right)$ describe the "Personality Analysis of Candidates" and consider the parameters $\mathrm{A}=\{\mathrm{e} 1, \mathrm{e} 3, \mathrm{e} 4, \mathrm{e} 5, \mathrm{e} 7, \mathrm{e} 8\}$ 
And

$$
\begin{aligned}
& F_{\mu}\left(e_{1}\right)=\left\{\left(\frac{c_{1}}{0.6}, 0.4\right),\left(\frac{c_{2}}{0.3}, 0.7\right),\left(\frac{c_{3}}{0.5}, 0.4\right),\left(\frac{c_{4}}{0.5}, 0.3\right),\left(\frac{c_{5}}{0.3}, 0.7\right),\left(\frac{c_{6}}{0.7}, 0.3\right),\left(\frac{c_{7}}{0.4}, 0.2\right),\left(\frac{c_{8}}{0.2}, 0.8\right)\right\}, \\
& F_{\mu}\left(e_{3}\right)=\left\{\left(\frac{c_{1}}{0.3}, 0.5\right),\left(\frac{c_{2}}{0.6}, 0.5\right),\left(\frac{c_{3}}{0.7}, 0.5\right),\left(\frac{c_{4}}{0.2}, 0.6\right),\left(\frac{c_{5}}{0.5}, 0.4\right),\left(\frac{c_{6}}{0.4}, 0.7\right),\left(\frac{c_{7}}{0.6}, 0.7\right),\left(\frac{c_{8}}{0.4}, 0.2\right)\right\}, \\
& F_{\mu}\left(e_{4}\right)=\left\{\left(\frac{c_{1}}{0.4}, 0.7\right),\left(\frac{c_{2}}{0.2}, 0.4\right),\left(\frac{c_{3}}{0.6}, 0.2\right),\left(\frac{c_{4}}{0.1}, 0.5\right),\left(\frac{c_{5}}{0.7}, 0.6\right),\left(\frac{c_{6}}{0.2}, 0.4\right),\left(\frac{c_{7}}{0.8}, 0.3\right),\left(\frac{c_{8}}{0.5}, 0.7\right)\right\}, \\
& F_{\mu}\left(e_{5}\right)=\left\{\left(\frac{c_{1}}{0.4}, 0.3\right),\left(\frac{c_{2}}{0.5}, 0.2\right),\left(\frac{c_{3}}{0.7}, 0.8\right),\left(\frac{c_{4}}{0.7}, 0.2\right),\left(\frac{c_{5}}{0.4}, 0.5\right),\left(\frac{c_{6}}{0.1}, 0.6\right),\left(\frac{c_{7}}{0.5}, 0.6\right),\left(\frac{c_{8}}{0.3}, 0.4\right)\right\}, \\
& F_{\mu}\left(e_{7}\right)=\left\{\left(\frac{c_{1}}{0.6}, 0.1\right),\left(\frac{c_{2}}{0.5}, 0.3\right),\left(\frac{c_{3}}{0.6}, 0.3\right),\left(\frac{c_{4}}{0.8}, 0.5\right),\left(\frac{c_{5}}{0.8}, 0.2\right),\left(\frac{c_{6}}{0.3}, 0.5\right),\left(\frac{c_{7}}{0.2}, 0.4\right),\left(\frac{c_{8}}{0.5}, 0.6\right)\right\}, \\
& F_{\mu}\left(e_{8}\right)=\left\{\left(\frac{c_{1}}{0.4}, 0.6\right),\left(\frac{c_{2}}{0.5}, 0.6\right),\left(\frac{c_{3}}{0.6}, 0.7\right),\left(\frac{c_{4}}{0.4}, 0.3\right),\left(\frac{c_{5}}{0.8}, 0.5\right),\left(\frac{c_{6}}{0.2}, 0.8\right),\left(\frac{c_{7}}{0.5}, 0.6\right),\left(\frac{c_{8}}{0.5}, 0.4\right)\right\},
\end{aligned}
$$

$$
\begin{aligned}
& G_{\delta}\left(\neg e_{1}\right)=\left\{\left(\frac{c_{1}}{0.3}, 0.6\right),\left(\frac{c_{2}}{0.5}, 0.2\right),\left(\frac{c_{3}}{0.4}, 0.5\right),\left(\frac{c_{4}}{0.2}, 0.4\right),\left(\frac{c_{5}}{0.6}, 0.4\right),\left(\frac{c_{6}}{0.3}, 0.7\right),\left(\frac{c_{7}}{0.6}, 0.3\right),\left(\frac{c_{8}}{0.7}, 0.2\right)\right\}, \\
& G_{\delta}\left(\neg e_{3}\right)=\left\{\left(\frac{c_{1}}{0.5}, 0.3\right),\left(\frac{c_{2}}{0.4}, 0.8\right),\left(\frac{c_{3}}{0.3}, 0.8\right),\left(\frac{c_{4}}{0.6}, 0.5\right),\left(\frac{c_{5}}{0.4}, 0.6\right),\left(\frac{c_{6}}{0.5}, 0.4\right),\left(\frac{c_{7}}{0.4}, 0.7\right),\left(\frac{c_{8}}{0.6}, 0.7\right)\right\}, \\
& G_{\delta}\left(\neg e_{4}\right)=\left\{\left(\frac{c_{1}}{0.5}, 0.7\right),\left(\frac{c_{2}}{0.7}, 0.3\right),\left(\frac{c_{3}}{0.4}, 0.2\right),\left(\frac{c_{4}}{0.3}, 0.8\right),\left(\frac{c_{5}}{0.3}, 0.5\right),\left(\frac{c_{6}}{0.6}, 0.5\right),\left(\frac{c_{7}}{0.2}, 0.4\right),\left(\frac{c_{8}}{0.3}, 0.5\right)\right\}, \\
& G_{\delta}\left(\neg e_{5}\right)=\left\{\left(\frac{c_{1}}{0.6}, 0.2\right),\left(\frac{c_{2}}{0.4}, 0.7\right),\left(\frac{c_{3}}{0.3}, 0.7\right),\left(\frac{c_{4}}{0.2}, 0.5\right),\left(\frac{c_{5}}{0.6}, 0.2\right),\left(\frac{c_{6}}{0.7}, 0.5\right),\left(\frac{c_{7}}{0.3}, 0.6\right),\left(\frac{c_{8}}{0.6}, 0.5\right)\right\}, \\
& G_{\delta}\left(\neg e_{7}\right)=\left\{\left(\frac{c_{1}}{0.4}, 0.8\right),\left(\frac{c_{2}}{0.5}, 0.4\right),\left(\frac{c_{3}}{0.3}, 0.5\right),\left(\frac{c_{4}}{0.2}, 0.7\right),\left(\frac{c_{5}}{0.1}, 0.8\right),\left(\frac{c_{6}}{0.7}, 0.2\right),\left(\frac{c_{7}}{0.6}, 0.5\right),\left(\frac{c_{8}}{0.4}, 0.6\right)\right\}, \\
& G_{\delta}\left(\neg e_{8}\right)=\left\{\left(\frac{c_{1}}{0.6}, 0.2\right),\left(\frac{c_{2}}{0.4}, 0.6\right),\left(\frac{c_{3}}{0.3}, 0.6\right),\left(\frac{c_{4}}{0.5}, 0.3\right),\left(\frac{c_{5}}{0.2}, 0.3\right),\left(\frac{c_{6}}{0.5}, 0.8\right),\left(\frac{c_{7}}{0.4}, 0.8\right),\left(\frac{c_{8}}{0.3}, 0.7\right)\right\} .
\end{aligned}
$$

The $b_{i}\left(e_{k}\right)$ of the bipolar possibility fuzzy soft set $\left(\mathrm{F} \mu, G_{\delta}, \mathrm{A}\right)$ is given in Table 1.

TABLE 1 . The $b_{i}\left(e_{k}\right)$ of the bipolar possibility fuzzy soft set $\left(\mathrm{F} \mu, G_{\delta}, \mathrm{A}\right)$.

\begin{tabular}{ccccccc}
\hline & $\mathrm{e} 1$ & $\mathrm{e} 3$ & $\mathrm{e} 4$ & $\mathrm{e} 5$ & $\mathrm{e} 7$ & $\mathrm{e} 8$ \\
\hline $\mathrm{c} 1$ & 0.24 & 0.15 & 0.28 & 0.12 & 0.06 & 0.24 \\
$\mathrm{c} 2$ & 0.21 & 0.30 & 0.08 & 0.10 & 0.15 & 0.30 \\
$\mathrm{c} 3$ & 0.20 & 0.35 & 0.12 & 0.56 & 0.18 & 0.42 \\
$\mathrm{c} 4$ & 0.15 & 0.12 & 0.05 & 0.14 & 0.40 & 0.12 \\
$\mathrm{c} 5$ & 0.21 & 0.20 & 0.42 & 0.20 & 0.16 & 0.40 \\
$\mathrm{c} 6$ & 0.21 & 0.28 & 0.08 & 0.06 & 0.15 & 0.16 \\
$\mathrm{c} 7$ & 0.08 & 0.42 & 0.24 & 0.30 & 0.08 & 0.30 \\
$\mathrm{c} 8$ & 0.16 & 0.08 & 0.35 & 0.12 & 0.30 & 0.20 \\
\hline
\end{tabular}

The $d_{i}\left(\neg e_{k}\right)$ of the bipolar possibility fuzzy soft set $\left(\mathrm{F} \mu, G_{\delta}, \mathrm{A}\right)$ is given in Table 2 . 
TABLE 2. The $d_{i}\left(\neg e_{k}\right)$ of the bipolar possibility fuzzy soft set $\left(\mathrm{F} \mu, G_{\delta}, \mathrm{A}\right)$.

\begin{tabular}{ccccccc}
\hline & $\mathrm{e} 1$ & $\mathrm{e} 3$ & $\mathrm{e} 4$ & $\mathrm{e} 5$ & $\mathrm{e} 7$ & $\mathrm{e} 8$ \\
\hline $\mathrm{c} 1$ & 0.18 & 0.15 & 0.35 & 0.12 & 0.32 & 0.12 \\
$\mathrm{c} 2$ & 0.10 & 0.32 & 0.21 & 0.28 & 0.20 & 0.24 \\
$\mathrm{c} 3$ & 0.20 & 0.24 & 0.08 & 0.21 & 0.15 & 0.18 \\
$\mathrm{c} 4$ & 0.08 & 0.30 & 0.24 & 0.10 & 0.14 & 0.15 \\
$\mathrm{c} 5$ & 0.24 & 0.24 & 0.15 & 0.12 & 0.08 & 0.06 \\
$\mathrm{c} 6$ & 0.21 & 0.20 & 0.30 & 0.35 & 0.14 & 0.40 \\
$\mathrm{c7}$ & 0.18 & 0.28 & 0.08 & 0.18 & 0.30 & 0.32 \\
$\mathrm{c} 8$ & 0.14 & 0.42 & 0.15 & 0.30 & 0.24 & 0.21 \\
\hline
\end{tabular}

According to Table 1, we have the positive information comparison table in Table 3.

TABLE 3. The positive information comparison.

\begin{tabular}{lcccccccc}
\hline & $\mathrm{c} 1$ & $\mathrm{c} 2$ & $\mathrm{c} 3$ & $\mathrm{c} 4$ & $\mathrm{c5}$ & $\mathrm{c} 6$ & $\mathrm{c7}$ & $\mathrm{c} 8$ \\
\hline $\mathrm{c} 1$ & 6 & 3 & 2 & 4 & 1 & 4 & 2 & 4 \\
$\mathrm{c} 2$ & 3 & 6 & 1 & 4 & 2 & 6 & 3 & 3 \\
$\mathrm{c} 3$ & 4 & 5 & 6 & 5 & 4 & 5 & 4 & 4 \\
$\mathrm{c} 4$ & 2 & 2 & 1 & 6 & 1 & 2 & 2 & 3 \\
$\mathrm{c5}$ & 5 & 5 & 2 & 5 & 6 & 5 & 4 & 5 \\
$\mathrm{c} 6$ & 2 & 3 & 1 & 4 & 2 & 6 & 2 & 2 \\
$\mathrm{c7}$ & 4 & 4 & 2 & 4 & 2 & 4 & 6 & 3 \\
$\mathrm{c} 8$ & 3 & 3 & 2 & 3 & 1 & 4 & 3 & 6 \\
\hline
\end{tabular}

According to Table 2, we have the negative information comparison table in Table 4.

TABLE 4. The negative information comparison.

\begin{tabular}{lllllllll}
\hline & $\mathrm{c} 1$ & $\mathrm{c} 2$ & $\mathrm{c} 3$ & $\mathrm{c} 4$ & $\mathrm{c5}$ & $\mathrm{c} 6$ & $\mathrm{c7}$ & $\mathrm{c8}$ \\
\hline $\mathrm{c} 1$ & -6 & -3 & -2 & -4 & -4 & -2 & -3 & -3 \\
$\mathrm{c} 2$ & -3 & -6 & -5 & -5 & -5 & -2 & -3 & -2 \\
$\mathrm{c} 3$ & -4 & -1 & -6 & -4 & -4 & -2 & -3 & -1 \\
$\mathrm{c} 4$ & -2 & -1 & -2 & -6 & -4 & -2 & -2 & -1 \\
$\mathrm{c5}$ & -3 & -1 & -3 & -2 & -6 & -2 & -2 & -2 \\
$\mathrm{c} 6$ & -4 & -4 & -4 & -5 & -4 & -6 & -4 & -4 \\
$\mathrm{c7}$ & -4 & -3 & -4 & -4 & -4 & -2 & -6 & -3 \\
$\mathrm{c} 8$ & -3 & -4 & -5 & -5 & -5 & -2 & -3 & -6 \\
\hline
\end{tabular}

The positive information score table is given in Table 5.

TABLE 5. The positive information scores.

\begin{tabular}{cccc}
\hline & Row sum & Column sum & Positive information score \\
\hline c1 & 26 & 29 & -3 \\
c2 & 28 & 31 & -3 \\
c3 & 37 & 17 & 20 \\
c4 & 19 & 35 & -16 \\
c5 & 37 & 19 & 18 \\
c6 & 22 & 36 & -14 \\
c7 & 29 & 26 & 3 \\
c8 & 25 & 30 & -5 \\
\hline
\end{tabular}

The negative information score table is given in Table 6 . 
TABLE 6. The negative information scores.

\begin{tabular}{cccc}
\hline & Row sum & Column & Negative information score \\
\hline c1 & -27 & -29 & 2 \\
c2 & -31 & -23 & -8 \\
c3 & -25 & -31 & 6 \\
c4 & -20 & -35 & 15 \\
c5 & -21 & -36 & 15 \\
c6 & -35 & -20 & -15 \\
c7 & -30 & -26 & -4 \\
c8 & -33 & -22 & -11 \\
\hline
\end{tabular}

The final score table is given in Table 7.

TABLE 7. The final score.

\begin{tabular}{cccc}
\hline & Positive information score & Negative information score & Final score \\
\hline c1 & -3 & 2 & -1 \\
c2 & -3 & -8 & -11 \\
c3 & 20 & 6 & 26 \\
c4 & -16 & 15 & -1 \\
c5 & 18 & 15 & 33 \\
c6 & -14 & -15 & -29 \\
c7 & 3 & -4 & -1 \\
c8 & -5 & -11 & -16 \\
\hline
\end{tabular}

It is concluded that the maximal score is 33 , hence $\mathrm{c} 5$ is the optimal choice object and so $\mathrm{c} 5$ is the best candidate for the position. In case that $\mathrm{c} 5$ cannot join the position, $\mathrm{c} 3$ will be selected.

\section{CONCLUSION}

The concept of bipolar possibility fuzzy soft sets is proposed and some operations, such as extended union, extended intersection, restricted union, restricted intersection, "AND", "OR", on the theory of bipolar possibility fuzzy soft sets are defined. Then a few of basic properties are studied. Finally, an application of bipolar possibility fuzzy soft sets with a general algorithm is given to solve decision making problems.

\section{ACKNOWLEDGMENTS}

Cong Men received a master degree from the Northwest Normal University in 2014, and the lecturer title from Lanzhou City University in 2017.

Bo Hai is currently an associate professor at Lanzhou City University.

Hailing Yao received his master degree from Lanzhou Jiao tong University. He is currently pursuing his Ph.D. degree (since 2016) at the Northwest Normal University.

\section{REFERENCES}

1. S. Alkhazaleh, A. R. Saleh, and N. Hassan, Soft multisite theory, Applied Mathematical Sciences, vol.5, no. 72, pp. 3561-3573, 2011.

2. S. Alkhazaleh, A. R. Saleh, and N. Hassan, Fuzzy parameterized interval-valued fuzzy soft set, Applied Mathematical Sciences, vol. 5, no. 67, pp. 3335-3346, 2011.

3. S. Alkhazaleh, A. R. Saleh, and N. Hassan, Possibility fuzzy soft set, Advances in Decision Sciences, Article ID 479756, 18 pages, 2011.

4. M. Salam, S. Abdullah, K. Ulla. Bipolar Fuzzy Soft sets and its applications in decision making problem [J]. Computer Science, 2013, 27(2):729-742.

5. K. T. Atanassov, Intuitionistic fuzzy sets, Fuzzy Sets and Systems, vol. 20, no. 1, pp. 87-96, 1986.

6. D. Dubois, H. Pride, An introduction to bipolar representations of information and preference, Int. J. Intel. Sys. 23(2008) 866-865. 
7. W.L. GAO, D.J. Boehner, Vague sets, IEEE Trans. Syst., Man Cyber. 23 (2) (1993) 610-614.

8. B. V. Gnedenko, the Theory of Probability, Chelsea, New York, NY, USA, 1962.

9. M. B. Gorzalzany, A method of inference in approximate reasoning based on interval-valued fuzzy sets, Fuzzy Sets and Systems, vol. 21, no. 1, pp. 1-17, 1987.

10. K.M. Lee, Bipolar-valued fuzzy sets and their basic operations, Proceeding International Conference, Bangkok, Thailand, (2000), 307-317.

11. P. K. Majid, R. Biswas, and A. R. Roy, Soft set theory, Computers and Mathematics with Applications, vol. 45, no. 4-5, pp. 555-562, 2003.

12. P. K. Majid, A. R. Roy, and R. Biswas, an application of soft sets in a decision-making problem, Computers and Mathematics with Applications, vol. 44, no. 8-9, pp. 1077-1083, 2002.

13. P. K. Majid, R. Biswas, and A. R. Roy, Fuzzy soft sets, Journal of Fuzzy Mathematics, vol. 9, no. 3, pp. 589602, and 2001.

14. D. Molotov, Soft set theory-first results, Computers \& Mathematics with Applications, vol. 37, no. 4-5, pp. 1931, 1999.

15. Z. Pawley, Rough sets, Int. J. Inform. Compute. Sci. 11 (1982) 341-356.

16. A. R. Roy and P. K. Majid, A fuzzy soft set theoretic approach to decision making problems, Journal of Computational and Applied Mathematics, vol. 203, no. 2, pp. 412-418, 2007.

17. M. Shabbier, M. Nazi. On Bipolar Soft Sets [J]. Mathematics, 2013.

18. L. A. Zaiden, Fuzzy sets, Information and Computation, vol. 8, pp. 338-353, 1965.

19. Y. Zoo and Z. Xiao, Data analysis approaches of soft sets under incomplete information, Knowledgebase Systems, vol. 21, no. 8, pp. 941-945, 2008. 